\title{
Surgical Treatment of Neonatal Giant Omphalocele in Delivery Room: A 10-Year Retrospective Study in East China
}

\author{
Fang Bin Shao ${ }^{1}$, Xiao Zhou Bao ${ }^{1}$, Jun Wang ${ }^{2}$, Zhong Rong $\mathrm{Li}^{1}$ and Li Bin Zhu ${ }^{1}{ }^{1,}{ }^{*}$ \\ ${ }^{1}$ The Second Affiliated Hospital and Yuying Children's Hospital, Wenzhou Medical University, Wenzhou, China \\ ${ }^{2}$ Xinhua Hospital Affiliated to Shanghai Jiaotong University, Shanghai, China \\ "Corresponding author: The Second Affiliated Hospital and Yuying Children's Hospital, Wenzhou Medical University, Wenzhou, China. Email: wz.zhulibin@aliyun.com \\ Received 2019 May 11; Revised 2019 June 30; Accepted 2019 August 09.
}

\begin{abstract}
Background: The survival rate of neonatal giant omphaloceles has improved over the past 10 years, but the mortality rate remains at approximately 50\% with traditional treatment. Delivery room surgery refers to a surgical procedure that is performed in the operating room to correct the structural defects of a newborn immediately after birth.

Objectives: This study aimed to investigate the application of delivery room surgery for giant omphaloceles in East China.

Methods: Neonates meeting the inclusion criteria of having omphalocele defects larger than $5 \mathrm{~cm}$ or of having most of the liver protruding into the sac were enrolled in this retrospective study. Data were evaluated specifically to determine the role of delivery room surgery in reducing the mortality of newborns with giant omphaloceles (GOs) and to identify factors that could optimize the conditions for traditional operations. A total of 52 newborns with GOs from two tertiary centres of paediatric surgery were identified. The patients were divided into two groups, the delivery room surgery group (group D) and the traditional operation group (group T), based on the perinatal management and time of surgery.

Results: There was a significantly higher survival rate in the group $\mathrm{D}(77.8 \%)$ than in the group T (50\%). The duration of ventilator use was $8.87 \pm 10.12$ days in the group D and $9.31 \pm 7.75$ days in the group T. The time of starting enteral nutrition was $12.88 \pm 4.19$ days in the group D and $16.75 \pm 8.59$ days in the group T. Newborns in the group T took more time to reach full enteral feeding. However, we did not find a significant difference in a comparison of the hospitalization time between the two groups (25.1 \pm 18.1 days in the group D and $22.4 \pm 11.1$ days in the group $\mathrm{T}$ ).

Conclusions: The strategy of delivery room surgery appears to improve the survival of newborns with GOs and reduce family burden. A further reduction in mortality rates will depend on promoting the application of the delivery room surgical procedure.
\end{abstract}

Keywords: Giant Omphalocele, Delivery Room Surgery, Surgical Outcome, Mortality

\section{Background}

Omphalocele is one of the most common abdominal wall defects caused by a failure of the abdominal internal organs to return to the abdominal cavity during embryonic development, and its incidence in neonates is approximately $1 / 4000$ - 6000 (1). When the abdominal wall defect exceeds $5 \mathrm{~cm}$ or when most of the liver is protruding into the sac, it is considered to be giant omphalocele (GO) (2). Due to the difference in the size of the tissue and the degree of abdominal dysplasia, the management and treatment of giant omphaloceles is difficult. Early treatment can improve the prognosis and reduce the burden on the mother and the family.

According to the China birth defect prevention and control report (2012) statistics, there are approximately 16 million newborns in China every year, and the incidence of birth defects is approximately 5.6\%. Every year, there are approximately 900,000 children born with birth defects in China, and the trend is increasing yearly. Among the children born with birth defects, approximately 35\% die after birth, and $40 \%$ suffer from lifelong disability; thus, along with lifelong birth defects, there is also a heavy spiritual and economic burden placed on the families and on society. According to an earlier study in China, the overall incidence of omphalocele is 15.2 per 10000 live birth and its overall perinatal mortality rate is approximately $51 \%$ (3). Improving the prognosis of children with birth defects is a top priority for us. Delivery room surgery refers to surgery that corrects structural defects immediately after birth, including foetal assessment, preoperative preparation, intraoperative collaboration, postoperative management and postoperative follow-up. In developed countries, the development of delivery room surgery has caused 
congenital malformations such as omphalocele and gastroschisis to be treated in time, thus reducing the mortality associated with such malformations. Currently, delivery room surgery has been successfully applied in clinical practice in China, and delivery room surgery is often implemented in the treatment of giant omphaloceles.

\section{Objectives}

This article reviews the treatment experiences of the past 10 years from China and discusses their development value.

\section{Methods}

The research data were obtained from two tertiary centres of paediatric surgery. The giant omphalocele cases of the Second Affiliated Hospital and Yuying Children's Hospital of Wenzhou Medical University and Xinhua Hospital Affiliated to Shanghai Jiaotong University during the past 10 years were retrospectively analysed. The delivery room surgery group (group D) and the traditional operation group (group T) were divided according to the perinatal management and time of surgery. The long-term cooperation and exchanges between two hospitals enable them to share the same philosophy of diagnosis, treatment and postoperative management. Before we start the study, we have an exclusion criterion that the child who had low Apgar scoring, broken sac or sepsis is excluded.

We collected data on all children treated in these two centres from 2007 to 2017 and established a unified giant omphalocele inclusion criteria of an abdominal wall defect with a size $>5 \mathrm{~cm}$ or with the liver within the sac. Data were collected on the gestational age, mode of delivery, birth weight, gender, operative plan, survival rate, length of ventilator use, time of starting enteral nutrition, and hospitalization time.

Data are presented as absolute values (n), frequencies $(\%)$, mean \pm SD. The data were statistically analyzed with SPSS18.0 statistical software. The Fisher's exact-test or chisquare test was used to compare proportions. The comparison between the unpaired groups with respect to continuous variables were performed with Student's $t$-test. A P value below 0.05 was considered statistically significant.

\subsection{Therapeutic}

All patients in the group D had a prenatal diagnosis of omphalocele before birth, and the mother of the child completed delivery through a caesarean section at one of the two centers. This process required the collaboration of an obstetrician, a neonatologist, an anaesthesiologist,
2 neonatal surgeons, and nurses. First, the obstetrician completed the delivery process. The child was placed in an overhead warmer after birth in the delivery room, and a venous access and a gastric tube were established. Then, the neonatologist and neonatal surgeon jointly assessed whether the child could tolerate the primary repair. If the child was unable to undergo a primary repair, the Silo bag was immediately placed, and delayed repair was necessary. If the child was eligible for the primary repair, warm saline gauze was used to protect the invaded organs and transport them to a next-door operating room. At this time, an anaesthesiologist should have completed the general anaesthesia and monitored blood pressure, heart rate, oxygen saturation, body temperature and so on. We can see a large mass in the umbilical region of the child (Figure 1A). The operation proceeded with reduction and primary fascial closure. Postoperative children were transferred to the NICU for unified monitoring and treatment. Two days after the sugery, we examined the incision of the child (Figure 1B).

Group T cases included children who were transferred to both centres after birth at an external hospital and who were born and received treatment at the two centres. The children who were born at an external hospital were transferred to the NICU after birth, and the neonatal surgeon and neonatologist jointly evaluated the condition of the child and completed the venous access, preoperative examination, and retention of the gastric tube. Then, the child was transferred to the operating room if primary repair was deemed safe. If the child was unable to undergo a primary repair, we placed a Silo bag and delayed the repair as was necessary. After the operation, the NICU physician continued to monitor the child.

\section{Results}

There were 36 cases in the group D and 16 cases in the group T. The gestational age, mode of delivery, birth weight, gender, operative plan, survival rate, length of ventilator use, time of starting parenteral nutrition and length of hospital stay were analysed. As shown in Table 1 , we analysed the patient demographics of these cases and found no significant difference in the gestational age, mode of delivery, birth weight, gender difference, and operative plan between the two groups ( $\mathrm{P}>0.05)$; thus, the data from the two groups were comparable. The male/female ratio was approximately 1:1.17. However, we analysed the survival rates of the two groups and found that the survival rate of the children in the group $\mathrm{D}(77.8 \%)$ was higher than that of the group $\mathrm{T}$ (50\%); the difference was statistically significant $(\mathrm{P}<0.05)$. 

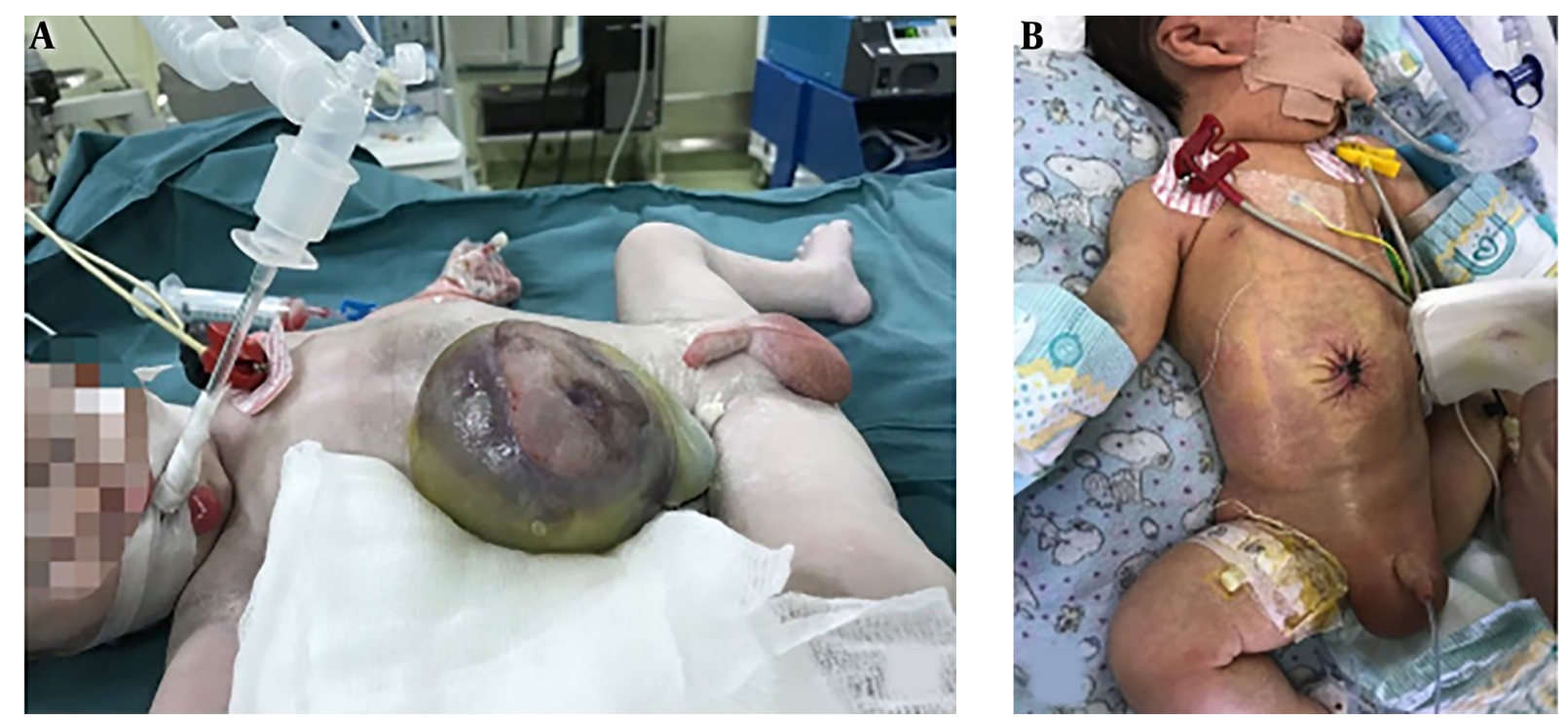

Figure 1. Perioperative umbilical photo of the child with giant omphalocele. A, umbilical mass before delivery room surgery; B, umbilical wound after delivery room surgery.

\begin{tabular}{|c|c|c|c|}
\hline Variables & Group $D(N=36)$ & Group $T(N=16)$ & P Value \\
\hline Gestational age, wk & $38.04 \pm 0.66$ & $37.58 \pm 1.18$ & 0.163 \\
\hline Mode of delivery, ${ }^{\text {a }}$ & & & 0.308 \\
\hline Cesarean & 36 & 15 & \\
\hline Vaginal & 0 & 1 & \\
\hline Birth weight, $g$ & $3084.9 \pm 568$ & $3129.7 \pm 595.5$ & 0.7973 \\
\hline Male/female ratio & $17 / 19$ & $7 / 9$ & 0.817 \\
\hline \multicolumn{4}{|l|}{ Operative plan } \\
\hline Primary repair & 30 & 12 & \\
\hline Staged repair & 6 & 4 & \\
\hline $\begin{array}{l}\text { Primary repair rate, } \\
\%\end{array}$ & 83.3 & 75 & 0.482 \\
\hline Survival & 28 & 8 & \\
\hline Death/abandon & 8 & 8 & \\
\hline Survival ratio, \% & 77.8 & 50 & 0.0452 \\
\hline
\end{tabular}

We analysed the duration of ventilator use, the time of starting parenteral nutrition, and the length of the hospital stay, and we compared the differences in the postoperative recovery of these children. As shown in Table 2, the duration of ventilator use ( $8.87 \pm 10.12$ days) in the group $\mathrm{D}$ were less than those in the group $\mathrm{T}(12.88 \pm 4.19$ days), and the difference was statistically significant $(P<0.05)$. Moreover, we found that the time of starting enteral nutri- tion (9.31 \pm 7.75 days) in the group D was earlier than that in the group T (16.75 \pm 8.59 days), and the difference was statistically significant $(\mathrm{P}<0.05)$. However, there was no significant difference between the two groups regarding the length of the hospital stay $(\mathrm{P}>0.05)$.

\begin{tabular}{lccc}
\hline \multicolumn{3}{l}{ Table 2. The Recovery Condition of Two Groups After Operation } & \\
\hline Variables & Group D (N= 36) & Group T (N=16) & P Value \\
\hline Ventilator use time, $\mathbf{d}$ & $8.87 \pm 10.12$ & $12.88 \pm 4.19$ & 0.015 \\
$\begin{array}{l}\text { Time on start enteral } \\
\text { nutrition, } \mathbf{d}\end{array}$ & $9.31 \pm 7.75$ & $16.75 \pm 8.59$ & 0.0187 \\
$\begin{array}{l}\text { Length of hospital } \\
\text { stay, } \mathbf{d}\end{array}$ & $25.1 \pm 18.1$ & $22.4 \pm 11.1$ & 0.5843 \\
\hline
\end{tabular}

As shown in Table 3, we analysed the causes of death/abandonment in the two groups and found that the overall mortality rate was approximately $30.8 \%$, resulting in an overall survival rate of $69.2 \%$. Among the children in the group D, 4 patients had respiratory failure (11.1\%), which was the main death/abandonment factor for the group D. Six patients (37.5\%) had a family burden in the group $\mathrm{T}$, which was the main death/abandonment factor in the group T.

\section{Discussion}

Despite the survival rate of neonatal omphalocele is higher in developed countries, the mortality rate of neonatal in China is still very high. In recent years, prenatal ultrasound for the diagnosis of abdominal wall deformity 


\begin{tabular}{lccc}
\hline \multicolumn{4}{l}{ Table 3. The Causes of Death/Abandonment in Two Groups } \\
\hline Variables & Group D $(\mathbf{N}=\mathbf{3 6})$ & Group T (N=16) & Total (N= 52) \\
\hline Mortality & $8(22.2)$ & $8(50)$ & $16(30.8)$ \\
$\begin{array}{l}\text { Respiratory } \\
\text { failure }\end{array}$ & $4(11.1)$ & $0(0.0)$ & $4(7.7)$ \\
Cardiac failure & $1(2.8)$ & $0(0.0)$ & $1(1.9)$ \\
\hline Sepsis & $0(0.0)$ & $1(6.25)$ & $1(1.9)$ \\
\hline DIC & $0(0.0)$ & $1(6.25)$ & $1(1.9)$ \\
\hline ACS & $1(2.8)$ & $0(0.0)$ & $1(1.9)$ \\
\hline Asphyxia & $1(2.8)$ & $0(0.0)$ & $1(1.9)$ \\
IVH & $1(2.8)$ & $0(0.0)$ & $1(1.9)$ \\
\hline Family burden & $0(0.0)$ & $6(37.5)$ & $6(11.5)$ \\
\hline
\end{tabular}

Abbreviations: ACS, abdominal compartment syndrome; DIC, disseminated intravascular coagulation; IVH, intraventricular hemorrhage.

${ }^{\mathrm{a}}$ Values are expressed as No. (\%).

has played an important role, especially in the distinction between omphalocele and gastroschisis. Due to both the numerical and structural chromosome anomalies that are known to be present in $30 \%-40 \%$ of pregnancies with foetal omphalocele (4), when there is a prenatal ultrasound diagnosis of omphalocele, the child needs to undergo amniocentesis to test for chromosomal abnormalities, such as trisomies 13 and 18 (5). At present, the principal treatment for giant omphalocele is to return the abdominal contents and close the abdominal wall defect when the children has been stabilized medically with good supportive care (6). For children who cannot undergo the primary repair or staged repair, a stage II repair is a good option, but the treatment of ventral hernia caused by this procedure is challenging for surgeons (7). In children with omphalocele, the development of the abdominal cavity is dysplastic; therefore, an early closure of the abdominal wall will lead to a sudden increase in abdominal pressure and will reduce the volume of the lungs, resulting in respiratory failure, heart failure, and abdominal compartment syndrome. In particular, respiratory failure is more common in neonates with giant omphaloceles because neonates with GO are more likely to have high risk factors for respiratory failure, such as pulmonary dysplasia (8).

When the abdominal wall defect is more than $5 \mathrm{~cm}$ or the sac contains a protruding liver, it is defined as GO in our study. However, the definition of GO is still controversial at present (9). Danzer et al. (8) defined GO as a large umbilical cord defect covered by membrane, containing most of the liver ( $>75 \%)$. In this study, we analysed the cases of giant omphalocele at two medical centres over the past 10 years, comparing the differences between the delivery room surgery and the routine operation. From the general information on the included cases, there was no sig- nificant difference in the gestational age, gender and birth weight of the two groups.

Previous studies comparing delayed with early closure for GO have found that an aggressive surgical approach (primary repair) in infants with GO is a safe option (10). Two central treatment teams were trained according to the basic principles of omphalocele treatment, so there was no significant difference in the proportion of primary repairs ( $82.1 \%$ vs. $75 \%$ ) between the two groups. The overall survival ratio was approximately $69.2 \%$; however, after comparing the two groups, we found that the survival ratio of the group D (77.8\%) was significantly higher than that of the group $\mathrm{T}(50 \%)$. If we do not take family burden into account, respiratory failure (7.7\%) was the main death/abandonment factor in both groups. It is worth noting that for the death/abandonment of the child, the mortality rate as a result of family burden was (11.5\%) higher than that as a result of respiratory failure, and it was concentrated in the group T. The reasons may be as follows: (1) The family members of the group T had no relevant knowledge about GO and its treatment before birth, so they lacked confidence in the treatment; whereas the family members of the group $\mathrm{D}$ had a deeper understanding of the disease after the doctor's explanation and had a more positive recognition of the doctor's technology and the prognosis of GO; (2) during the early stages of respiratory failure, sepsis and other complications, some parents in the group $\mathrm{T}$ worried about the high cost of the treatment and tended to choose to terminate the treatment; (3) some of the children in the group T came from families with economic burdens and were transferred from the grass root hospitals. Children with giant omphalocele not only bring spiritual and economic burden to their parents but also experience follow-up problems. In the study of Hijkoop et al. (11), they followed up with children with omphalocele who survived until they were 2-years-old and found that most of the children (over 80\%) with giant omphalocele had delayed motor development.

The most significant difference between the delivery room surgery and the traditional surgery group is that the repair occurs earlier. Acorroding an early study, it has been reported that patients with GO are less likely to undergo management with early closure compared with small omphalocele (12). In a study of omphalocele by Na et al. (13), the immediate repair (IR group) was defined as undergoing surgery immediately after birth (usually less than 10 minutes), and after 3 hours of life, the surgical repair was defined as a late repair (control group). The children in the IR group started enteral nutrition earlier, had a lower infection rate, and had a shorter hospitalization time than those in the control group. Because of the substantial abdominal wall defect and the risk of postoperative respira- 
tory failure (which is related to the small thoracic profile of the newborn, pulmonary dysplasia, and possibly even ventilator injury) in GO children, postoperative management of these children is quite difficult (6). We routinely used assisted ventilation after surgery in the two groups. If the ventilator could be removed as soon as possible, it could ensure better recovery after surgery. We recorded the postoperative recovery condition of the two groups of children, including the duration of ventilator use, the time of starting enteral nutrition, and the length of hospital stay. Comparing the duration of ventilator use of the two groups, we found that the duration of ventilator use (days) in the group D was significantly less than that in the group $\mathrm{T}$, which further reduced the risk of ventilator-related injuries such as respiratory failure and ventilator-related infections. In addition, we found that enteral nutrition was started earlier in children in the group D, indicating that the recovery of intestinal function in the group D occurred earlier than it did in the group $\mathrm{T}$, which helped to reduce complications related to intravenous nutrition. The faster removal of the ventilator and earlier start of enteral nutrition not only means that the postoperative recovery of the group D was faster than that of the group $\mathrm{T}$ but also shows that the delivery room surgery had greater advantages and improved the developmental prospects more than the conventional surgery. The reasons may be as follows: (1) delivery room surgery does not require excessive transport processes, which allows for the removal of the structural abnormalities of the children as soon as possible, thus preventing disease progression and reducing the infection rate; (2) delivery room surgery can avoid excessive intestinal flatulence caused by crying and making noise after birth. The abdominal pressure after early closure of the abdominal wall defect is relatively low, which is conducive to the early removal of the ventilator and recovery of intestinal function after surgery; (3) the early repair and treatment of the delivery room surgery can alleviate the psychological and economic burden for family. However, we did not find significant differences in the comparison of the hospitalization time between the two groups. The reasons may be as follows: (1) All of the children included in the study were diagnosed with GO, so the treatment and postoperative management of these children were more difficult, and the length of the hospital stay was longer than for minor/medium omphalocele; (2) some children in the group $\mathrm{T}$ were discharged due to economic factors, which led to a shorter hospital stay in this group.

\subsection{Conclusions}

In summary, we want to emphasize that delivery room surgery has many advantages in the treatment of GO and is a valuable tool. We believe that the results of this study are important to conceive when counseling parents following a prenatal diagnosis of omphalocele, since the strategy of delivery room surgery appears to improve the survival of newborns with GOs and reduce family burden. We hope that these data can help more children's medical centres to utilize delivery room surgery and can encourage more families to receive delivery room surgery.

\section{Acknowledgments}

We would like to thank our colleagues from the Department of Pediatric Surgery for their assistance with data collection.

\section{Footnotes}

Authors' Contribution: Study concept and design: Li Bin Zhu, Jun Wang, and Zhong Rong Li; analysis and interpretation of data: Fang Bin Shao; drafting of the manuscript: Fang Bin Shao; critical revision of the manuscript for important intellectual content: Fang Bin Shao, Li Bin Zhu, and Xiao Zhou Bao; statistical analysis: Fang Bin Shao and Li Bin Zhu.

Conflict of Interests: The authors declared no conflict of interests related to the subject matter or material discussed in the manuscript.

Ethical Approval: This study was approved by the Ethics Committee of the Second Affiliated Hospital and Yuying Children's Hospital of Wenzhou Medical University.

Funding/Support: No funding was received for this study.

\section{References}

1. Bauman B, Stephens D, Gershone H, Bongiorno C, Osterholm E, Acton $\mathrm{R}$, et al. Management of giant omphaloceles: A systematic review of methods of staged surgical vs. nonoperative delayed closure. J Pediatr Surg. 2016;51(10):1725-30. doi: 10.1016/j.jpedsurg.2016.07.006. [PubMed: 27570242].

2. Kruit AS, Al-Ani SA, Jester I, Jester A. Multilayered flap technique: A method for delayed closure of giant omphalocele. Ann Plast Surg. 2016;76(6):680-3. doi: 10.1097/SAP.0000000000000589. [PubMed: 26207550].

3. Zhou GX, Liang J, Zhu J, Dai L, Wang YP, Miao L. [An epidemiological study on omphalocele in China during 1996 to 2000]. Zhonghua Yu Fang Yi Xue Za Zhi. 2004;38(5):328-30. Chinese. [PubMed: 15498248].

4. Conner P, Vejde JH, Burgos CM. Accuracy and impact of prenatal diagnosis in infants with omphalocele. Pediatr Surg Int. 2018;34(6):62933. doi: 10.1007/s00383-018-4265-x. [PubMed: 29637257]. [PubMed Central: PMC5954074].

5. Hsu CC, Lin SP, Chen CH, Chi CS, Lee HC, Hung HY, et al. Omphalocele and gastroschisis in Taiwan. Eur J Pediatr. 2002;161(10):552-5. doi: 10.1007/s00431-002-1031-8. [PubMed: 12297903]. 
6. Akinkuotu AC, Sheikh F, Olutoye OO, Lee TC, Fernandes CJ, Welty SE, et al. Giant omphaloceles: Surgical management and perinatal outcomes. J Surg Res. 2015;198(2):388-92. doi: 10.1016/j.jss.2015.03.060. [PubMed: 25918004].

7. Ojha S, Parashar S, Doshi D, Bansal RK. Giant omphalocele complicated by postoperative duodenal obstruction. APSP J Case Rep. 2017;8(1):5. doi: 10.21699/ajcr.v8i1.518. [PubMed: 28164002]. [PubMed Central: PMC5253613].

8. Danzer E, Victoria T, Bebbington MW, Siegle J, Rintoul NE, Johnson MP, et al. Fetal MRI-calculated total lung volumes in the prediction of short-term outcome in giant omphalocele: Preliminary findings. Fetal Diagn Ther. 2012;31(4):248-53. doi: 10.1159/000334284. [PubMed: 22572017].

9. Whitehouse JS, Gourlay DM, Masonbrink AR, Aiken JJ, Calkins CM, Sato TT, et al. Conservative management of giant omphalocele with topical povidone-iodine and its effect on thyroid function. J Pediatr Surg. 2010;45(6):1192-7. doi: 10.1016/j.jpedsurg.2010.02.091. [PubMed:
20620319].

10. Rijhwani A, Davenport M, Dawrant M, Dimitriou G, Patel S, Greenough A, et al. Definitive surgical management of antenatally diagnosed exomphalos. J Pediatr Surg. 2005;40(3):516-22. doi: 10.1016/j.jpedsurg.2004.11.028. [PubMed: 15793728].

11. Hijkoop A, Peters NCJ, Lechner RL, van Bever Y, van Gils-Frijters APJM, Tibboel D, et al. Omphalocele: From diagnosis to growth and development at 2 years of age. Arch Dis Child Fetal Neonatal Ed. 2019;104(1):F1823. doi: 10.1136/archdischild-2017-314700. [PubMed: 29563149].

12. Vachharajani AJ, Rao R, Keswani S, Mathur AM. Outcomes of exomphalos: An institutional experience. Pediatr Surg Int. 2009;25(2):13944. doi: 10.1007/s00383-008-2301-y. [PubMed: 19066916].

13. Na Q, Liu C, Cui H, Zhang Z, Yin S, Li Q. Immediate repair compared with delayed repair of congenital omphalocele: Short-term neonatal outcomes in China. J Int Med Res. 2011;39(6):2344-51. doi: 10.1177/147323001103900634. [PubMed: 22289553]. 